\title{
Comparação entre treinamento concorrente e corrida em piscina funda associados à orientação nutricional na perda de peso e composição corporal de indivíduos obesos
}

\author{
Comparison between concurrent training and running in deep water associated with \\ nutritional counseling on weight loss and body composition in obese individuals
}

\author{
Lisete Griebeler Souza', Thiago Rozales Ramis², Luciane Cabral de Fraga ${ }^{3}$, Jerri Luiz Ribeiro ${ }^{4}$, \\ Zilda Elizabeth de Albuquerque Santos ${ }^{5}$
}

${ }^{1}$ Nutricionista, Centro Universitário Metodista IPA, Porto Alegre, RS; ${ }^{2}$ Educador Físico, Mestrando em Reabilitação e Inclusão do Centro Universitário Metodista IPA Porto Alegre, RS; ${ }^{3}$ Educadora Física, Mestra em Reabilitação e Inclusão pelo Centro Universitário Metodista do IPA, Porto Alegre, RS; ${ }^{4}$ Educador Físico, Doutor em Ciências do Movimento Humano. Professor titular e Coordenador do Programa de Pós Graduação em Reabilitação e Inclusão no Centro Universitário Metodista IPA, Porto Alegre, RS; ${ }^{5}$ Nutricionista, Doutora em Medicina e Ciências da Saúde. Professora Adjunta do Departamento de Medicina Interna da Faculdade de Medicina da Universidade Federal do Rio Grande do Sul (UFRGS), Porto Alegre, RS.

\section{RESUMO}

Objetivos: Comparar os efeitos de dois programas de exercícios, treinamento concorrente e corrida em piscina funda, associados à orientação nutricional, na perda de peso e composição corporal de obesos.

Métodos: Cinquenta indivíduos foram divididos em dois grupos, conforme o tipo de programa de treinamento físico. Um dos grupos, composto por 23 indivíduos, foi submetido a um treinamento concorrente (aeróbico e força); e 27 foram submetidos a corrida em piscina funda (aeróbico). Todos receberam a mesma orientação nutricional. Os participantes foram avaliados quanto ao peso, altura, circunferências, índice de massa corporal e composição corporal (massa gorda e massa magra), antes e após 45 dias do programa. Para análise das variáveis entre os grupos foi aplicado o teste $t$ de Student e para avaliar o efeito da perda de peso nas demais variáveis, a correlação linear de Pearson. O nível de significância adotado foi de menos de 0,05 .

Resultados: A idade média dos participantes foi 48,1 \pm 12 anos, sendo 42 (84\%) do gênero feminino. Após 45 dias de intervenção, 42 indivíduos perderam em média $2,96 \%$ do peso inicial, com redução significativa em todas as variáveis antropométricas, exceto da massa magra. Não houve diferença significativa na perda de peso e na composição corporal entre os indivíduos que praticaram o treinamento concorrente e os que praticaram corrida em piscina funda.

Conclusões: Os dois grupos submetidos a treinamento físico e intervenção nutricional apresentaram perda de peso e alteração positiva na composição corporal, sem predomínio de efeito entre os tipos de exercício.

DESCRITORES: OBESIDADE; COMPOSIÇÃO CORPORAL; EDUCAÇÃO NUTRICIONAL; ATIVIDADE FÍSICA.

\section{ABSTRACT}

\begin{abstract}
Aims: To compare the effects of two exercise programs, concurrent training and running in deep water, associated with nutritional counseling, in weight loss and body composition in obese individuals.

Methods: Fifty subjects were divided into two groups according to the type of physical training program. One group, composed of 23 individuals, underwent a concurrent training (aerobic and strength); and 27 underwent deep water running (aerobic). All received the same nutritional counseling. Participants were assessed for weight, height, girth, body mass index and body composition (fat mass and lean mass) before and after 45 days of the program. Student's $t$ test was applied for analysis of variables between groups and Pearson correlation test was used to evaluate the effect of weight loss in the other variables. The significance level was set at less than 0.05 .

Results: The mean age of participants was $48.1 \pm 12$ years and $42(84 \%)$ were female. After 45 days of intervention, 42 individuals lost a medium of $2.96 \%$ of initial weight, with significant reductions in all anthropometric variables, except the lean mass. There was no significant difference in weight loss and body composition among individuals who practiced concurrent training or practiced running in deep water. Conclusions: Both groups that underwent physical training and nutritional intervention showed weight loss and a positive change in body composition, without predominant effect between the exercise types.
\end{abstract}

KEY WORDS: OBESITY; BODY COMPOSITION; NUTRITIONAL EDUCATION; PHYSICAL ACTIVITY. 


\section{INTRODUÇÃO}

A obesidade e o sobrepeso são definidos como um acúmulo de gordura corporal, que pode prejudicar a saúde. Segundo a Organização Mundial de Saúde (OMS), desde 1980 a prevalência de obesidade aumentou mais do que o dobro no mundo inteiro. ${ }^{1}$ No Brasil, dados da Pesquisa de Orçamento Familiar (POF 2008-2009), realizada pelo IBGE, mostraram que 50\% dos homens e $48 \%$ das mulheres estão com excesso de peso e, destes, $12,4 \%$ dos homens e $16,9 \%$ das mulheres estão obesos. ${ }^{2}$ Em Porto Alegre, os resultados encontrados pela pesquisa VIGITEL (2011), ${ }^{3}$ do Ministério da Saúde, foram mais elevados que os da média nacional da POF, estando $55,4 \%$ dos adultos com excesso de peso e destes, $19,6 \%$ com obesidade.

A obesidade, considerada uma enfermidade crônica, frequentemente está associada a doenças cardiometabólicas, musculoesqueléticas e neoplasias. ${ }^{4,5} \mathrm{~A}$ distribuição da gordura em diferentes compartimentos corporais interfere no risco à saúde, principalmente a gordura visceral, sobretudo em função do seu efeito deletério na sensibilidade à insulina. ${ }^{6,7}$ As causas da obesidade são complexas e envolvem múltiplos e interligados fatores, tais como ambiente, estilo de vida, emoções e material genético. Aumento do consumo calórico, diminuição do gasto energético ou as duas situações simultaneamente, contribuem decisivamente para um desequilíbrio no balanço energético, que se reflete no aumento de peso. ${ }^{6,8}$

As complicações clínicas, potencialmente graves e limitantes da qualidade de vida, associadas à obesidade, bem como o aumento crescente da proporção de indivíduos obesos no mundo todo, tornam a obesidade um importante problema de saúde pública. Desenvolver estratégias, simples e viáveis, para combater esta pandemia, torna-se uma prioridade. Este estudo teve como objetivo avaliar alterações no peso e na composição corporal de indivíduos obesos submetidos à modificação do estilo de vida, através de orientação nutricional e dois tipos de treinamento físico.

\section{MÉTODOS}

\section{Formação dos grupos de estudo}

O presente estudo, caracterizado como uma intervenção não controlada, foi realizado no Núcleo de Pesquisa do Centro Universitário Metodista - IPA, no período de agosto de 2011 a agosto de 2012. A amostra foi composta por indivíduos da comunidade, recrutados através dos meios de comunicação, que atenderam os critérios de inclusão: adultos, de ambos os gêneros, com índice de massa corporal (IMC) $\geq 25 \mathrm{~kg} / \mathrm{m}^{2}$. Foram excluídos indivíduos em uso de medicação para perda de peso, que haviam sido submetidos a cirurgia bariátrica no último ano, em uso corticoterapia, em tratamento para distúrbios da tireoide, gestantes e nutrizes. O estudo foi aprovado pelo Comitê de Ética do Centro Universitário Metodista - IPA, sob o protocolo CEP n ${ }^{\circ} 396 / 2011$. Todos os participantes assinaram o Termo de Consentimento Livre e Esclarecido.

Os indivíduos foram avaliados por uma nutricionista em dois momentos: antes do início do programa e aos 45 dias do mesmo. Na primeira consulta foi realizada anamnese alimentar, avaliação antropométrica e exame de bioimpedância. Nesse momento todos receberam orientações nutricionais e estímulo à mudança nos hábitos alimentares. As orientações alimentares foram baseadas no Guia Alimentação Saudável Para Todos, do Ministério da Saúde ${ }^{9}$ e na I Diretriz Brasileira de Diagnóstico e Tratamento da Síndrome Metabólica - I DBSM. ${ }^{10}$

No mesmo dia da primeira consulta com a nutricionista, os participantes foram separados em dois grupos, homogêneos quanto à idade, sexo, peso, altura e IMC, para receber treinamento físico orientado por educador físico. No primeiro grupo ("grupo concorrente") ficaram 23 indivíduos, que receberam treinamento concorrente (exercício aeróbio e de força), e no segundo grupo ("grupo piscina") ficaram 27 indivíduos, que receberam treinamento de corrida em piscina funda (exercício aeróbio).

Ao final de 45 dias, em novo encontro com a nutricionista, foi realizado um inquérito Recordatório de 24 horas, ${ }^{11}$ com o objetivo de avaliar a adesão à orientação nutricional. Nesse encontro foram refeitas as medidas antropométricas e o exame de bioimpedância.

\section{Anamnese alimentar}

Para conhecimento e avaliação dos hábitos alimentares no início do programa, foi aplicada uma adaptação simplificada da História Alimentar, inquérito dietético utilizado na prática clínica na primeira consulta, com o objetivo de conhecer os hábitos alimentares do paciente, que é capaz de estimar a ingestão habitual qualitativa. ${ }^{11} \mathrm{O}$ hábito alimentar foi comparado ao preconizado pela pirâmide alimentar adaptada. ${ }^{12}$

\section{Avaliação antropométrica}

Foram medidos peso, altura e circunferências da cintura (CC), do quadril (CQ) e do pescoço (CP). O 
peso foi medido em balança antropométrica mecânica da marca Filizola (Porto Alegre, RS), com capacidade para $150 \mathrm{~kg}$, de acordo com a recomendação do SISVAN.$^{13}$ Os indivíduos foram pesados descalços e com roupas leves. A estatura foi verificada no mesmo momento, utilizando-se a régua antropométrica acoplada à balança, estando o indivíduo posicionado ereto, imóvel, com os pés juntos e os braços estendidos ao longo do corpo, a cabeça erguida e livre de adereços, olhando para um ponto fixo na altura dos olhos. Os IMC foi calculado pela divisão do peso em quilos pelo quadrado da altura em metros, e foi classificado de acordo com a OMS (1995). ${ }^{14}$

As medidas das circunferências foram realizadas com os indivíduos em pé, utilizando uma fita antropométrica da marca Wiso (Florianópolis, SC). A medida da $\mathrm{CC}$ foi realizada no ponto entre a crista ilíaca ântero-superior e a última costela, segundo a I DBSM. ${ }^{10}$ Os resultados foram classificados de acordo com os critérios do International Diabetes Federation - IDF, ${ }^{15}$ para indivíduos sul-americanos, onde: valores para homens maiores de $90 \mathrm{~cm}$ e para mulheres maiores de $80 \mathrm{~cm}$ são considerados risco aumentado de doença cardiovascular. A medida da CQ foi realizada no local de maior proeminência dos glúteos. A razão cintura/ quadril (RCQ) foi calculada dividindo-se o valor da CC pela $C Q$, sendo considerado risco cardiovascular valores $\geq 1,0$ para homens e $\geq 0,80$ para mulheres. ${ }^{16} \mathrm{~A}$ $\mathrm{CP}$ foi realizada no ponto médio, ao nível da cartilagem cricotireóidea, segundo Martin et al. ${ }^{17}$ Em homens esta medida é realizada logo abaixo do pomo de Adão. Esta medida é indicativa de sobrepeso se o resultado encontrado for $\geq 37 \mathrm{~cm}$ para homens ou $\geq 34 \mathrm{~cm}$ para mulheres, ou indicativa de obesidade se o resultado encontrado for $\geq 39,5 \mathrm{~cm}$ para homens ou $\geq 36,5 \mathrm{~cm}$ para mulheres. ${ }^{18}$

\section{Exame de bioimpedância}

Para a análise da composição corporal por meio do exame de bioimpedância foi utilizado o analisador Biodynamics BIA 310. No momento do teste todos estavam em jejum de pelo menos 4 horas; não realizaram atividades físicas extenuantes nas 24 horas anteriores; urinaram pelo menos 30 minutos antes; não ingeriram bebidas alcoólicas nas 48 horas anteriores, não utilizaram medicamentos diuréticos nos 7 dias que antecederam o teste e, no caso das mulheres, não estavam no período menstrual. Os dados coletados foram percentual de gordura corporal (GC), peso da massa gorda (MG), peso da massa magra (MM), Taxa Metabólica Basal (TMB) e quantidade de água corporal total (litros). O percentual de gordura corporal é considerado elevado se $>25 \%$ para homens e $>30 \%$ para mulheres, pontos de corte propostos pela National Institutes of Health - NHLBI. ${ }^{19}$

\section{Treinamento físico}

O treinamento físico foi realizado em 24 sessões, com quatro sessões semanais (um total de seis semanas, ou 45 dias). Antes do início de cada modalidade de treinamento, os participantes foram familiarizados com os equipamentos e o ambiente de treino.

O treinamento concorrente foi composto pela associação de caminhada orientada com treinamento de força. Nas sessões eram realizados 5 minutos de aquecimento, 30 minutos de exercício aeróbio e 30 minutos de treinamento de força. Foram executados oito exercícios primários e complementares, ${ }^{20}$ adaptados de McCarthy et al. ${ }^{21}$ Os intervalos entre as séries e exercícios foram aproximadamente de 60 segundos. A ordem de execução e cadência (2 segundos para cada fase; concêntrica e excêntrica) foram mantidas durante todo o treinamento.

O treinamento de corrida em piscina funda foi realizado com colete flutuador em uma piscina semiolímpica (os indivíduos não encostavam os pés no fundo da piscina). A duração da sessão foi semelhante à do treinamento concorrente e a intensidade foi monitorada através da escala de Borg. ${ }^{22}$

Para o treinamento concorrente foi realizada uma progressão linear, na qual a cada duas semanas a intensidade dos exercícios de força e aeróbio progrediam, variando de 50\% de uma repetição máxima (1 RM) (15 repetições por série) e $50 \%$ do volume máximo de oxigênio $\left(\mathrm{VO}_{2}\right.$ máx) (11 a 12 na escala de Borg) até $70 \%$ de 1 RM (8 repetições) e $70 \%$ do $\mathrm{VO}_{2}$ máx (14 a 15 na Escala de Borg). ${ }^{23}$ Para o treinamento de corrida em piscina funda também foi realizada uma periodização linear com as mesmas progressões de intensidade do exercício aeróbio do treinamento concorrente.

\section{Análise estatística}

Os dados foram analisados utilizando o pacote estatístico SPSS (Statistical Package for Social Sciences) versão 18.0 para Windows. Para comparar os parâmetros antropométricos nos dois momentos do estudo (zero e 45 dias), foi aplicado o teste $t$ de Student para amostras pareadas. Para as comparações das variações entre os grupos, foi utilizado o teste $t$ de Student para amostras independentes. Para avaliar a associação entre os parâmetros antropométricos foi 
aplicado o teste de correlação linear de Pearson. O nível de significância adotado foi de $5 \%(\mathrm{p} \leq 0,05)$.

\section{RESULTADOS}

Participaram do estudo 50 indivíduos, com idade média de 48,1 \pm 12 anos, sendo $42(84 \%)$ do gênero feminino. No início do estudo os participantes apresentavam peso médio de $90,1 \pm 13,3 \mathrm{~kg}$ e IMC de $34,6 \pm 4,5 \mathrm{~kg} / \mathrm{m}^{2}$, que os classificava como obesos e com elevado risco cardiovascular, de acordo com CC $(108,3 \pm 9,6 \mathrm{~cm})$ e RCQ $(0,93 \pm 0,07)$. As mulheres apresentavam um percentual de gordura corporal maior que os homens e uma menor massa magra (Tabela 1).

Tabela 1. Características antropométricas da amostra de 50 indivíduos obesos, no início do estudo sobre os efeitos de programas de exercícios consistindo em treinamento concorrente ou corrida em piscina funda, associados à orientação nutricional. Centro Universitário Metodista - IPA, Porto Alegre, agosto de 2011 a agosto de 2012

\begin{tabular}{|lccc|}
\multicolumn{1}{c}{ Variável } & $\begin{array}{c}\text { Amostra } \\
\text { total } \\
\mathbf{n = 5 0}\end{array}$ & $\begin{array}{c}\text { Homens } \\
\mathbf{n = 8}\end{array}$ & $\begin{array}{c}\text { Mulheres } \\
\mathbf{n = 4 2}\end{array}$ \\
\hline Peso $(\mathrm{kg})$ & $90,1 \pm 13,3$ & $104,6 \pm 14,2$ & $87,3 \pm 11,4$ \\
\hline Índice de massa corporal $\left(\mathrm{kg} / \mathrm{m}^{2}\right)$ & $34,6 \pm 4,5$ & $35,2 \pm 5,2$ & $34,4 \pm 4,4$ \\
\hline Gordura corporal $(\%)$ & $38,3 \pm 4,5$ & $31,9 \pm 3,4$ & $39,5 \pm 3,5$ \\
\hline Gordura corporal $(\mathrm{kg})$ & $34,6 \pm 7,2$ & $33,7 \pm 8,1$ & $34,8 \pm 7,1$ \\
\hline Massa magra $(\mathrm{kg})$ & $54,5 \pm 8,5$ & $70,9 \pm 6,5$ & $52,1 \pm 4,9$ \\
\hline Circunferência da cintura $(\mathrm{cm})$ & $108,3 \pm 9,6$ & $117,3 \pm 12,3$ & $106,5 \pm 8,1$ \\
\hline Circunferência do quadril $(\mathrm{cm})$ & $116,2 \pm 8,2$ & $116,9 \pm 7,5$ & $116 \pm 8,4$ \\
\hline Relação cintura/quadril & $0,93 \pm 0,07$ & $1,00 \pm 0,04$ & $0,91 \pm 0,06$ \\
\hline Circunferência do pescoço $(\mathrm{cm})$ & $38,4 \pm 3,0$ & $42,1 \pm 3,3$ & $37,7 \pm 2,4$ \\
\hline
\end{tabular}

Analisando a amostra total, ao final da intervenção, oito indivíduos $(16 \%)$, todos do gênero feminino, não perderam peso e até ganharam em média $0,8 \mathrm{~kg}$ $(0,93 \%$ do peso inicial); e 42 indivíduos perderam em média $2,7 \mathrm{~kg}$ ( $2,96 \%$ do peso inicial). Os homens apresentaram maior perda de peso do que as mulheres $(3,2 \%$ e $2,3 \%$ respectivamente), porém esta diferença não foi significativa $(\mathrm{p}=0,336)$.

Entre os indivíduos que perderam peso, houve redução significativa em todas as variáveis antropométricas. Todos reduziram o percentual de GC $(p<0,001)$ e aumentaram a MM $(p=0,046)$. Nos indivíduos que ganharam peso, houve aumento significativo somente no peso corporal e IMC (Tabela 2).

Em uma análise mais detalhada do grupo que perdeu peso, observamos que quatro indivíduos $(8 \%$ da amostra) perderam mais do que $2,96 \%$ do peso inicial (em média 5\%). Este subgrupo, ao contrário dos demais, apresentou perda de MM significativa $(p=0,003)$. No grupo que perdeu peso, houve correlação positiva e significativa entre a perda de peso e a redução do percentual de GC, do IMC e das circunferências (Tabela 3).

Analisando os resultados, de acordo com o tipo de treinamento físico orientado durante o estudo, não houve diferença significativa na perda de peso corporal, redução no IMC, percentual de GC, circunferências e ganho de MM entre os grupos (Tabela 4).

Tabela 2. Variáveis antropométricas ao início e ao final do estudo, do grupo que perdeu e ganhou peso. Amostra constituída por 50 indivíduos obesos submetidos a programas de exercícios com treinamento concorrente ou corrida em piscina funda, associados à orientação nutricional. Centro Universitário Metodista - IPA, Porto Alegre, agosto de 2011 a agosto de 2012

\begin{tabular}{|c|c|c|c|c|c|c|c|}
\hline \multirow{2}{*}{ Variável } & \multicolumn{3}{|c|}{$\begin{array}{c}\text { Grupo Perda de Peso } \\
n=42(84 \%)\end{array}$} & \multicolumn{3}{|c|}{$\begin{array}{c}\text { Grupo Ganho de Peso } \\
n=8(16 \%)\end{array}$} & \multirow[t]{2}{*}{$\mathbf{p}^{+}$} \\
\hline & 0 dias & 45 dias & $\mathrm{p}^{*}$ & 0 dias & 45 dias & p* $^{*}$ & \\
\hline Peso (kg) & $91 \pm 13,3$ & $88,3 \pm 13,2$ & $<0,001$ & $85,1 \pm 13,5$ & $85,9 \pm 13,7$ & 0,005 & $<0,001$ \\
\hline Índice de massa corporal $\left(\mathrm{kg} / \mathrm{m}^{2}\right)$ & $34,9 \pm 4,4$ & $33,9 \pm 4,1$ & $<0,001$ & $32,6 \pm 4,5$ & $33 \pm 4,9$ & 0,041 & $<0,001$ \\
\hline Gordura corporal (\%) & $37,9 \pm 4,6$ & $35,5 \pm 5,3$ & $<0,001$ & $40,1 \pm 3,4$ & $39,3 \pm 3,7$ & 0,304 & 0,012 \\
\hline Gordura corporal (kg) & $34,6 \pm 7$ & $31,4 \pm 6,7$ & $<0,001$ & $34,5 \pm 8,7$ & $34,1 \pm 8,8$ & 0,576 & $<0,001$ \\
\hline Massa magra (kg) & $56,4 \pm 8,7$ & $56,9 \pm 9,6$ & 0,046 & $50,7 \pm 5,3$ & $51,7 \pm 5,2$ & 0,151 & 0,408 \\
\hline Circunferência da cintura $(\mathrm{cm})$ & $109,1 \pm 9,7$ & $106,8 \pm 9,2$ & $<0,001$ & $104 \pm 8,4$ & $104,2 \pm 9,2$ & 0,891 & 0,098 \\
\hline Circunferência do quadril (cm) & $116 \pm 7,9$ & $113 \pm 7,1$ & $<0,001$ & $117 \pm 10$ & $116,3 \pm 10,5$ & 0,270 & 0,048 \\
\hline Relação cintura/quadril & $0,94 \pm 0,06$ & $0,94 \pm 0,08$ & 0,132 & $0,89 \pm 0,06$ & $0,89 \pm 0,05$ & 0,802 & 0,723 \\
\hline Circunferência do pescoço $(\mathrm{cm})$ & $38,7 \pm 3,1$ & $37,5 \pm 3,0$ & $<0,01$ & $36,9 \pm 2$ & $36,6 \pm 2,3$ & 0,388 & 0,075 \\
\hline
\end{tabular}

* Teste t de Student pareado; ${ }^{\dagger}$ teste $t$ de Student independente. 
Tabela 3. Correlações entre perda de peso e variáveis analisadas. Amostra constituída por 50 indivíduos obesos submetidos a programas de exercícios com treinamento concorrente ou corrida em piscina funda, associados à orientação nutricional. Centro Universitário Metodista - IPA, Porto Alegre, agosto de 2011 a agosto de 2012

\begin{tabular}{|lcc|}
\multicolumn{1}{c}{ Variável } & \multicolumn{2}{c}{ Perda de peso } \\
\cline { 2 - 3 } & $\mathbf{r}^{*}$ & $\mathbf{p}$ \\
\hline Redução da gordura corporal $(\%)$ & 0,442 & 0,001 \\
\hline Redução do índice de massa corporal $\left(\mathrm{kg} / \mathrm{m}^{2}\right)$ & 0,960 & $<0,001$ \\
\hline Redução da circunferência da cintura $(\mathrm{cm})$ & 0,444 & 0,001 \\
\hline Redução da circunferência do quadril $(\mathrm{cm})$ & 0,351 & 0,012 \\
\hline Redução da circunferência do pescoço $(\mathrm{cm})$ & 0,277 & 0,051 \\
\hline Redução da relação cintura/quadril & 0,126 & 0,385 \\
\hline
\end{tabular}

* Coeficiente de correlação de Pearson.

Tabela 4. Comparação entre as variáveis do grupo que perdeu peso $(\mathrm{N}=42)$, conforme o tipo de atividade física realizada: corrida em piscina funda (aeróbico) ou treinamento concorrente (aeróbico e força). Estudo de indivíduos obesos submetidos a programas de exercícios associados à orientação nutricional, por 45 dias. Centro Universitário Metodista - IPA, Porto Alegre, agosto de 2011 a agosto de 2012

\begin{tabular}{|lccc|}
\hline \multicolumn{1}{c}{ Variável } & $\begin{array}{c}\text { Treinamento } \\
\text { aeróbico }\end{array}$ & $\begin{array}{c}\text { Treinamento } \\
\text { concorrente }\end{array}$ & p* \\
\hline Perda de peso $(\mathrm{kg})$ & $-2 \pm 2,1$ & $-2,4 \pm 2,2$ & 0,608 \\
\hline Perda de peso $(\%)$ & $-2,3 \pm 2,3$ & $-2,5 \pm 2,5$ & 0,719 \\
\hline $\begin{array}{l}\text { Redução do índice de } \\
\text { massa corporal }\left(\mathrm{kg} / \mathrm{m}^{2}\right)\end{array}$ & $-0,72 \pm 0,87$ & $-0,91 \pm 0,84$ & 0,437 \\
\hline $\begin{array}{l}\text { Perda de gordura }(\%) \\
\text { Ganho de massa magra }(\mathrm{kg})\end{array}$ & $-1,85 \pm 1,5$ & $-2,6 \pm 1,95$ & 0,131 \\
\hline $\begin{array}{l}\text { Redução da circunferência } \\
\text { da cintura }(\mathrm{cm})\end{array}$ & $-1,61 \pm 4,08$ & $-2,25 \pm 3,86$ & 0,573 \\
\hline $\begin{array}{l}\text { Redução da circunferência } \\
\text { do quadril }(\mathrm{cm})\end{array}$ & $-2,41 \pm 3,34$ & $-2,91 \pm 2,75$ & 0,566 \\
\hline $\begin{array}{l}\text { Redução da circunferência } \\
\text { do pescoço }(\mathrm{cm})\end{array}$ & $-1,14 \pm 1,18$ & $-0,86 \pm 1,26$ & 0,408 \\
\hline Relação cintura/quadril & $0,01 \pm 0,04$ & $0,01 \pm 0,03$ & 0,777 \\
\hline
\end{tabular}

* Teste t de Student independente.

\section{DISCUSSÃO}

No presente estudo, a intervenção nutricional associada ao exercício físico produziu efeito positivo em $84 \%$ dos participantes, que atingiram uma perda ponderal média de 2,96\% $(2,7 \mathrm{~kg})$ em apenas seis semanas de intervenção, com redução significativa em todas as variáveis antropométricas avaliadas. Franz et al. ${ }^{24} \mathrm{em}$ uma revisão sistemática que incluiu 80 estudos, com oito diferentes tipos de intervenções, combinados ou isolados, de dieta, exercício e medicamentos, encontraram que as intervenções que envolveram estratégias nutricionais promoveram a perda de 5 a $9 \%$ do peso inicial nos primeiros seis meses de seguimento.
O objetivo da perda de peso é reduzir percentual de GC e medidas antropométricas, sobretudo CC; mas deve-se considerar que perda de peso, dentro do preconizado para melhora cardiometabólica (no mínimo $5 \%$ do peso inícial), ${ }^{10}$ pode resultar também em redução da MM, e este é um resultado indesejado. A MM é um compartimento metabolicamente ativo e contribui para o gasto energético corporal, auxiliando na perda de peso e na manutenção deste, posteriormente. Neste estudo, a perda de peso de 2,97\% foi acompanhada de redução do percentual de GC e da CC, mas não da MM, satisfazendo o objetivo dos programas para melhorar a saúde dos indivíduos.

No entanto, os quatro participantes que perderam em média $5 \%$ do peso inicial apresentaram perda significativa de MM, sugerindo que uma redução muito rápida no peso pode aumentar a chance de redução na MM.

A literatura tem apresentado resultados interessantes em estudos que aliaram restrição calórica ao exercício físico. Monteiro et al., ${ }^{25}$ estudando 30 mulheres obesas no climatério, divididas em dois grupos (dieta e dieta hipocalórica + exercício), encontraram redução no peso, IMC e CC em ambos os grupos, porém com redução de peso e CC maiores no grupo exercício, levandoos a concluir que os resultados mais satisfatórios são atingidos quando se alia dieta hipocalórica e exercício aeróbico.

Da mesma forma, Valle et al., ${ }^{26}$ estudando $40 \mathrm{mu}$ lheres divididas em três grupos (exercício, exercício + dieta e dieta), encontraram redução significativa no peso, IMC e percentual de GC, além dos níveis séricos de colesterol total e triglicerídeos, em todos os grupos, mas com redução significativa na $\mathrm{MM}$ somente no grupo dieta. Pritchard et al., ${ }^{27}$ avaliando 58 homens com sobrepeso, divididos em três grupos (dieta, exercício e controle), encontraram perda de peso e redução na massa gorda nos grupos dieta e exercício; manutenção da MM no grupo exercício e perda de MM somente no grupo dieta. Esses estudos evidenciam que a associação entre exercícios e orientação nutricional auxilia na perda de peso e minimiza a perda de MM, assim como foi observado na maioria da população do presente estudo.

Barbato et al. ${ }^{28}$ encontraram, em quatro meses de orientação alimentar e exercício físico, perda de $7,8 \mathrm{~kg}$ (8,5\%) e redução na CC de $10,1 \mathrm{~cm}$ em $47 \%$ dos participantes do estudo, com correlação positiva entre a perda ponderal e a redução da circunferência da cintura ( $\mathrm{r} 0,75$ e $\mathrm{p}<0,001)$. No presente estudo, em apenas 45 dias de acompanhamento e com perda média de $2,7 \mathrm{~kg}(2,96 \%)$, observamos, em $84 \%$ dos participantes, 
correlação positiva e significativa entre perda ponderal e todas as variáveis antropométricas analisadas, com maior correlação com IMC e CC.

Alguns autores afirmam que o tipo de exercício influencia na composição corporal durante programas de perda de peso. É o caso de Hauser et al., ${ }^{29}$ que referem que os exercícios de resistência muscular combinados com o exercício aeróbico parecem alcançar melhores resultados, por auxiliar no aumento da taxa metabólica, manter e/ou aumentar a massa muscular e otimizar os índices de mobilização e utilização de gordura durante o emagrecimento.

Porém, os resultados do presente estudo não mostraram diferença significativa na redução do peso, IMC, percentual de GC e circunferências, bem como ganho de MM, nos indivíduos que praticaram atividade física aeróbica associada à de resistência (treinamento concorrente), quando comparados aos que praticaram atividade puramente aeróbica (corrida em piscina funda). Do mesmo modo, Fett et al., ${ }^{30}$ estudando apenas mulheres, encontraram redução significativa no peso, IMC e percentual de GC, sem alteração na MM, com dois tipos de exercício físico (resistido + aeróbico e somente aeróbico). Pavlou et al., ${ }^{3}$ encontraram perda de peso e massa gorda, sem modificação na MM, em 72 obesos submetidos a uma intervenção com dieta + exercício de força + aeróbico.

Independente da composição corporal ou do tipo de intervenção, a perda ponderal contribui para a redução do risco cardiovascular. Gordon et al. ${ }^{32}$ observaram efeito anti-hipertensivo com a perda ponderal, em apenas 12 semanas de intervenção (exercício aeróbico, dieta, exercício aeróbico + dieta), em indivíduos sedentários e com sobrepeso. Valle et al. ${ }^{33}$ encontraram melhora no perfil lipídico, em mulheres divididas em dois grupos: controle e dieta. O grupo dieta, após 12 semanas, apresentou redução significativa no peso, percentual de GC, IMC e perfil lipídico. Embora não tenhamos avaliado pressão arterial e perfil lipídico, nossos resultados mostraram correlação positiva entre perda de peso e $\mathrm{CC}$, esta última um marcador de risco cardiovascular. De acordo com a International Diabetes Federation, ${ }^{15}$ a CC reflete a gordura abdominal, que está associada a alterações metabólicas, como dislipidemias, intolerância à glicose e hipertensão arterial, fatores de risco cardiovascular. ${ }^{34}$

Reconhecemos o pequeno período de intervenção (45 dias) e o reduzido número da amostra (50 indivíduos), como limitações do nosso estudo. São necessárias pesquisas com períodos maiores de intervenção e número amostral maior, para que se possam confirmar os achados deste estudo.

Os resultados do estudo permitem concluir que intervenção nutricional associada a um programa de treinamento físico foi eficaz para perda de peso e produziu alteração favorável na composição corporal. Estes resultados foram independentes do tipo de exercício físico entre os programas testados nesta amostra, treinamento concorrente ou corrida em piscina funda.

\section{REFERÊNCIAS}

1. World Health Organization. Obesity. [Internet]. [cited 2011 Sep 24]. Available from: http://www.who.int/topics/obesity/es

2. Instituto Brasileiro de Geografia e Estatística Pesquisa de Orçamentos Familiares 2008-2009. Antropometria e Estado Nutricional de Crianças, Adolescentes e Adultos no Brasil. [Internet]. Rio de Janeiro: IBGE; 2010. [cited 2013 Aug 20]. Available from: http://www. ibge.gov.br/home/estatistica/populacao/condicaodevida/pof/2008_2009_encaa/pof_20082009_encaa.pdf

3. VIGITEL Brasil 2011. Vigilância de Fatores de Risco e Proteção para Doenças Crônicas Não Transmissíveis por meio de Inquérito Telefônico. [Internet]. [cited 2013 Aug 20]. Available from: http://portalsaude.saude.gov.br/portalsaude/arquivos/pdf/2012/Ago/22/ vigitel_2011_final_0812.pdf

4. World Health Organization Obesity and overweight. [Internet]. [cited 2013 Aug 20]. Available from: http://www.who.int/mediacentre/ factsheets/fs311/en/

5. Pinheiro ARO, Freitas SFT, Corso ACT. Uma abordagem epidemiológica da obesidade. Rev Nutr. 2004;17(4):523-33.

6. Associação Brasileira para o Estudo da Obesidade e da Síndrome Metabólica Diretrizes brasileiras de obesidade. $3^{\mathrm{a}}$ ed. [Internet]. [cited 2013Aug 20]. Available from: http://www.abeso.org.br/pdf/diretrizes_brasileiras_obesidade_2009_2010_1.pdf

7. Carneiro G, Faria AN, Ribeiro Filho FF, Guimarães A, Lerário D, Ferreira SRG, Zanella MT.Influência da Distribuição da Gordura Corporal sobre a Prevalência de Hipertensão Arterial e Outros Fatores de Risco Cardiovascular em Indivíduos Obesos. Rev Assoc Med Bras. 2003;49(3):306-11.

8. Francischi RPP, Pereira LO, Freitas CS, Klopfer M, Santos RG, Vieira P, Lancha Júnior AH. Obesidade: atualização sobre sua etiologia, morbidade e tratamento. Rev Nutr. 2000;13(1):17-28.

9. Brasil. Ministério da Saúde. Alimentação Saudável Para Todos. [Internet]. [cited 2013 Jul 20]. Available from: http://189.28.128.100/ nutricao/docs/geral/10passosAdultos.pdf 
10. I Diretriz Brasileira de Diagnóstico e Tratamento da Síndrome Metabólica. Arq Bras Cardiol. 2005;84(1):1-28.

11. Fisberg RM, Martini LA, Slater B. Métodos de Inquéritos Alimentares. In: Fisberg RM, Marchioni DML, Martini LA. Inquéritos Alimentares: métodos e bases científicos. São Paulo: Manole; 2005.

12. Philippi ST, Latterza AR, Cruz ATR, Ribeiro LC. Pirâmide Alimentar Adaptada: Guia para escolha dos alimentos. Rev Nutr. 1999;12(1): 65-80.

13. Brasil. Ministério da Saúde. Sistema de Vigilância Alimentar e Nutricional. Vigilância alimentar e nutricional: orientações básicas para a coleta, processamento, análise de dados e informação em serviços de saúde. [Internet]. Brasília: Ministério da Saúde; 2004 [cited 2013 Sep 05]. Available from: http://189.28.128.100/nutricao/docs/geral/orientacoes_basicas_sisvan.pdf

14. World Health Organization Physical Status: the use and interpretation of anthropometry. [Internet]. Geneva: Technical Report Series 854; 1995 [cited 2013 Sep 05]. Available from: http://www.who.int/childgrowth/publications/physical_status/en/

15. International Diabetes Federation. The IDF consensus worldwide definition of the Metabolic Syndrome. [Internet]. Belgium: International Diabetes Federation, 2006 [cited 2013 Sep 05]. Available from: http://www.idf.org/webdata/docs/MetSyndrome_FINAL.pdf

16. Yusuf S, Hawken S, Ônpuu S, Bautista L, Franzosi MG, Commerford P, Lang CC, Rumboldt Z, Onen CL, Lisheng L, Tanomsup S, Wangai Jr P, Razak F, Sharma AM, Anand SS. Obesity and the risk of myocardial infarction in 27,000 participants from 52 countries: a case-control study. Lancet. 2005;366(9497):1640-9.

17. Martin SE, Mathur R, Marshall I, Douglas NJ. The effect of age, sex, obesity and posture on upper airway size. The European Respiratory Journal. 1997;10(9):2087-90.

18. Ben-Noun L, Sohar E, Laor A. Neck circumference as a simple screening measure for identifying overweight and obese patients. Obesity Research. 2001;9(8):470-7.

19. National Heart, Lung and Blood Institute Clinical Guidelines on the Identification, Evaluation and Treatment of Overweight and Obesity in Adults. [Internet]. NIH Publication. 1998 [cited 2013 Sept 9]. Available from: http://www.nhlbi.nih.gov/guidelines/obesity/ob_gdlns.pdf

20. Fleck SJ, Kraemer WJ. Fundamentos do treinamento de força muscular. 3. Ed. Porto Alegre: Artmed; 2006.

21. Mccarthy JP et al. Compatibility of adaptive responses with combining strength and endurance training. Med Sci Sports Exerc. 1995;27(3):429-36.

22. Borg G. Perceived Exertion and Pain Scales. Human Kinectics. 1988.

23. American College of Sports Medicine. ACSM's Guidelines for exercise testing and Prescription. $8^{\text {a }}$ ed. Baltimore: The point; 2009.

24. Franz MJ, VanWormer JJ, Crain AL, Boucher JL, Histon T, Caplan W, Bowman JD, Pronk NP. Weight-loss outcomes: a systematic review and meta-analysis of weight-loss clinical trials with a minimum 1-year follow-up. J Am Diet Assoc. 2007;107(10):1755-67.

25. Monteiro RCA, Riether PTA, Burini RC. Efeito de um Programa Misto de Intervenção Nutricional e Exercício Físico sobre a Composição Corporal e os Hábitos Alimentares de Mulheres Obesas em Climatério. Rev Nutr. 2004;17(4):479-89.

26. Valle VS, Mello DB, Fortes MSR, Dantas EHM, Mattos MA. Efeito da Dieta e do Ciclismo Indoor Sobre a Composição Corporal e Nível Sérico Lipídico. Arq Bras Cardiol. 2010;95(2):173-8.

27. Pritchard JE, Nowson CA, Wark JD. A worksite program for overweight middle-aged men achieves lesser weight loss with exercise than with dietary change. J Am Diet Assoc. 1997;97(1):37-42.

28. Barbato KBG, Martins RCV, Rodrigues MLG, Braga JU, Francischetti EA, Genelhu V. Efeitos da Redução de Peso Superior a 5\% nos Perfis Hemodinâmico, Metabólico e Neuroendócrino de Obesos Grau I. Arq Bras Cardiol. 2006;87(1):12-21.

29. Hauser C, Benetti M, Rebelo FPV. Estratégias para o emagrecimento. Rev Bras Cineantropom Desempenho Humano. $2004 ; 6(1): 72-81$.

30. Fett CA, Fett WCR, Oyama SR, Marchini S. Composição corporal e somatótipo de mulheres com sobrepeso e obesas pré e pós-treinamento em circuito ou caminhada. Rev Bras Med Esporte. 2006;12(1):45-50.

31. Pavlou KN, Steffee WP, Lerman RH, Burrows BA. Effects of dieting and exercise on lean body mass, oxygen uptake and strength. Med Sci Sports Exerc. 1985;17(4):466-71.

32. Gordon NF, Scott CB, Levine BD. Comparison of Single Versus Multiple Lifestyle Interventions: Are the Antihypertensive Effects of Exercise Training and Diet-Induced Weight Loss Additive?Am J Cardiol. 1997;79(6):763-7.

33. Valle VS, Biehl C, Mello DB, Fortes MSR, Dantas EHM. Efeito da dieta hipoenergética sobre a composição corporal e nível sérico lipídico de mulheres adultas com sobrepeso. Rev Nutr. 2010;23(6):959-67.

34. Rezende FAC, Rosado LEFPL, Ribeiro RCL, Vidigal FC, Vasques ACJ, Bonard IS, Carvalho CR. Índice de Massa Corporal e Circunferência Abdominal: Associação com Fatores de risco Cardiovascular. Arq Bras Cardiol. 2006; 87(6):728-34. 